\title{
Evaluation of Web Pages as a Tool in Public Relations
}

\author{
Peter Trkman and Jaka Lindic \\ University of Ljubljana, Faculty of Economics
}

peter.trkman@ef.uni-li.si jaka.lindic@ef.uni-li.si

\begin{abstract}
The paper deals with the question, how the efficiency and successfulness of web pages in information dissemination can be measured. The paper concentrates on the use of a corporate web page in public relations with different interest groups. Basic principles of public relations theory are summarized.

The importance of web-page credibility is emphasized and different factors that can have influence on it are identified. Obviously, reliability, punctuality and quality of published information are of utmost importance. However other factors that can affect credibility (such as technical quality, page structure etc.), are also studied.

Special attention is paid to another important topic in public relations theory - how can a web page be used to build and flourish dialogue relationships between the company and different interest groups that affect company's performance.

The newly developed model CUT (Content, Usability, Technology) for evaluation of web pages is presented. The model includes evaluation of web pages from different perspectives. While accurate, complete and up-to-date information is certainly crucial, other factors from usability and technology perspective are also emphasized. The model use is demonstrated on a case study of companies from different countries and industry branches.
\end{abstract}

Keywords: public relations, evaluation of web pages, usability, dissemination of information, interest groups.

\section{Introduction}

Public relations are becoming an increasingly important topic in today's competitive economy: how to efficiently inform different interest groups that can affect the performance of a company, is certainly a crucial question. Theory and practice have repeatedly shown that efficient communication with public can be vital for the survival and development of a company.

While a company can use different media for communication (television, radio, press, leaflets etc.) we have witnessed a tremendous development and commercialization of the Internet in the recent years. Consequently, nowadays almost every company has its own web page and updates it

Material published as part of this journal, either on-line or in print, is copyrighted by Informing Science. Permission to make digital or paper copy of part or all of these works for personal or classroom use is granted without fee provided that the copies are not made or distributed for profit or commercial advantage AND that copies 1) bear this notice in full and 2) give the full citation on the first page. It is permissible to abstract these works so long as credit is given. To copy in all other cases or to republish or to post on a server or to redistribute to lists requires specific permission from the publisher at Publisher@InformingScience.org more or less regularly. However, as shown in this and other papers, only few companies are using their web page effectively as part of their whole strategy.

A suitably developed web page can be an important addition in achieving this goal for a particular company. The 
obvious problem in designing a web page is the gap between developers and users ("web designers are not users" is a famous quote of the usability guru Jakob Nielsen). Usability studies are increasingly used to test suitability of web design and structure.

In order to attain the desired goal in the usage of web in public relations a company obviously needs good knowledge of both fields: public relations theory and its goals on one hand and usability and web page development concepts on the other. In this paper we connect those two fields of research. The main purpose of the paper is to present a new multi-criteria model for the evaluation of web pages. The model is called CUT, after its three main criteria: Content, Usability and Technology.

In the paper this model is specially tailored to the use of measuring the quality of web pages as a tool in public relations. Important guidelines that should be used in public relations are identified. Those topics include relationships building with different interest groups and credibility of published information and web page as a whole.

The presented model can serve as a framework for a particular company when developing, evaluating or re-designing its web page as it provides a comprehensive review of important topics that have to be considered when creating and maintaining a web page. It can also be used as a tool to compare the quality of different pages, either from the same branch or for cross-sectional (or cross-national) analyses.

An example is shown in the paper where a sample of 15 corporate web pages is examined, evaluated and compared. The general finding of this experiment is that, while there was certainly a considerable improvement in the overall quality in recent years, the general quality of web pages still leaves much to be desired. The presented model also allows us to point out the main deficiencies of a certain web page.

The structure of this paper is as follows: the first section presents the main concepts of public relations theory, along with the effect of Internet in this field. Then main methods and concepts of usability testing are shown. The third section presents the CUT model. The use of the model is finally demonstrated by an evaluation of a sample of corporate web pages from different countries and branches.

\section{Public Relations Theory}

Business success of a company increasingly depends on different stakeholders and successful communication and interaction between them and a company. Effective public relations with all relevant interest groups are widely recognized as an important tool for enabling effective communication and consequently prosperity of a company.

Esrock and Leichty (2000) identified 6 main interest groups, which a company should include in its public relations plan: customers, dealers, employees, prospective employees, press and investors. Traditional media (such as newspapers) usually have a limited space and scope and thus cannot provide the content in such a way that the specific interests of every group would be satisfied within a single medium. Therefore they usually target one interest group (one of the typical examples of this targeting is financial newspapers that mainly target investors, while information for other groups is limited).

On the other hand a web site can have a number of sections that target the unique wishes and needs of several audiences (Esrock \& Leichty, 1999). The importance of a specific interest group varies from site to site; the three audiences that are targeted most often are investors, prospective employees and customers. However most sites target different groups $-65 \%$ of the studied pages have specific contents for at least 3 of the above-mentioned groups (Esrock \& Leichty, 2000). Therefore it is not surprising that one of the general principles for building PR web pages is that a 
company should try to include information of general value for each of the mentioned publics (Kent \& Taylor, 1998).

Consequently, this paper does not concentrate on a specific interest group and the efficiency of communication with it. We provide a framework for the evaluation of web pages that can be used for a single interest group or for the evaluation of a web page as a whole.

Unsurprisingly, web pages have earned an increasingly important role in public relations strategy. The practicians most frequently cite the following anticipated advantages of including a web page as an additional channel for communication with public (Newland-Hill \& White, 2000):

- it shows competitiveness of the company

- it supplements media relations

- it helps reaching new audiences

- it builds relationships with interesting publics.

The users most often mention advantages like the scope and depth of published information and speed at which information can be retrieved, while reliability is the most important concern - as found with a survey of computer journalists in (Hachigian \& Hallahan, 2003).

Four possible models for public relations from the communication aspect were already presented 20 years ago in (Grunig, 1984):

- press agency: main purpose is propaganda, communication is one-way and complete truth is not essential,

- public information: main purpose is dissemination of information with one-way communication. Truth is important,

- two-way asymmetrical: communication goes in two ways, but with imbalanced effects; the main goal is scientific persuasion,

- two-way symmetrical communication: the main purpose is mutual understanding, communication goes in two ways with balanced effects.

A web site can be used as a tool in any of those 4 models - either for propaganda only (this can most easily be seen in various get-rich-quick or loss-weight-fast sites, although some other sites also follow this model), dissemination of information (many governmental and similar sites have this as their main purpose) or two-way asymmetrical communication (topics that are seen as important and crucial by the public are emphasized).

However, we believe that proactive companies with quality web pages should strive to achieve the fourth model - namely to build and maintain two-way symmetrical communication with its users. The most important difference between this one and the other 3 models is that in this model a company also changes its behavior according to the feedback of the interest groups. While it is possible to measure users feedback without a web page (with different surveys etc.), web pages offer an ideal way to gather much information about users, their habits and attitudes. Obviously this is only possible if a web page and its use in public relations is seen as an important complement to other efforts of a company not as an unnecessary addition.

The CUT model allows the measurement of the quality of web pages for each of the four models. However it is most suitable and important in the case of two-way symmetrical communication, as shown in the continuation of the paper.

As shown, web sites can have an important role in public relations - however the most important question is certainly which of the technical characteristics, web design solutions and content of a web site have the most influence on successfulness of a particular web site. Most theoretical and practical findings confirm that two most important topics are building of relationships with the 
users and credibility of web pages. Some considerations about those two topics are presented in the next section.

\section{Relationships Building}

Relationships' building is often emphasized as the most important task of public relations. Many authors even equal public relations and relationships building. Cutlip (Cutlip, Center, \& Broom, 1994, pp. 2), for example, defines PR as »the management function that establishes and maintains mutually beneficial relationships between an organization and the publics on whom its success or failure depends $\ll$.

A web site should assist a company in building close relationships with its stakeholders, because it offers the company an ideal opportunity to assist in building and maintaining close relationships with each of interest groups that were listed previously (customers, investors, press etc.)

Relationship building certainly requires a continuous effort and an efficient web page can only be part of the whole picture. However (with or without a web page) we believe that two most important components for relationships building are interpersonal communication (ideally, it should be two-way symmetrical as mentioned previously) and the quality of web page interaction.

\section{Interpersonal communication between users and the company}

Especially e-mail can be a vital tool to enable interpersonal communication. While every effort should be made to make the presentation and structure of a web page as clear and usable as possible, it is unavoidable that some users will have questions that are either not answered on the web page or the users were unable to find the published information. In both cases an important task is a prompt reply to all enquires of members of the interested publics.

Ideally, the response rate to users enquires should be close to $100 \%$. However the response rate of about $30 \%$ is found in practice (Kent, Taylor, \& White, 2003; Taylor, Kent, \& White, 2001)

Interpersonal communication can also be between different users. Community building can be especially important. Communities of users are important, because they can add content to the site that would otherwise be hard to add for web page creators. This content (e. g. user opinions) can be seen by other users as more trustworthy.

\section{Quality of web site interaction}

While interpersonal communication is a prerequisite for relationships building, the first impressions of the users towards a site can largely depend on the quality of web page interaction. The process of interaction should be as intuitive as possible for the user. The intuitiveness of interaction is more important than just the number of steps a user has to make to accomplish a certain task.

Sites should be dynamic enough to encourage all potential publics to explore them, information rich enough to meet the needs of very diverse publics, and interactive enough to allow users to pursue further informational issues and dialogic relationships. The final principle offers one more tool for fostering relationships (Kent \& Taylor., 1998). Quality of user forms (for feedback, comment, enquires) is also important. User forms must be without technical and logical mistakes and easy to access and use for the visitors of the site.

It should not be forgotten that ease of interface is believed to be a prerequisite for Webbed dialogue (Taylor et al., 2001).

The CUT model presented in the next sections allows the measurement of both components. Interpersonal communication (both the quality and speed of the response are measured) is included 
in the Content criterion, while quality of interaction (intuitiveness of the process and single page interaction) can be found in the Usability criterion. This separation is due to the fact that good communication between a user and the company can increase the value of the content for the user (or even add new content), while the quality of web page interaction increases the usability of the page as a whole.

Not surprisingly, content and user friendliness are also closely connected - a study of 100 Fortune 500 companies (Esrock, 1998) found that companies that develop more user-friendly pages also tend to develop content that goes beyond mere product information and financial materials. A study of activists organizations (Kent et al, 2003) came to a similar conclusion - pages with more communicative designs are also more likely to respond to user e-mail enquires.

\section{Credibility}

While one of the main advantages of the Internet is that it is democratic in nature and every company, an interest group or individual can post various content. Consequently we can say that World Wide Web is the first mass medium that can reach mass audience but the content is not filtered (White \& Raman, 2000). However this poses a serious question about credibility.

Since anyone can publish anything on the web, special attention has to be paid to credibility of a particular web page. Every company has to realize that credibility of a web page can also affect the credibility of the whole company (and vice versa).

The potential lack of credibility of web pages and the Internet as a whole can be illustrated with the findings of Middleberg/Ross Survey of Media in the Wired World (Middleberg \& Ross, 1999, 2000). While $98 \%$ of journalists are using web regularly, they consider credibility as one of the main problems - among the rated categories only trade associations sites are found more credible than not. Unsurprisingly, message boards and chat rooms are seen as least credible. Similarly Hachigian and Hallahan (2003) found that journalists are approaching web sites skeptically and that both reliability of published information and reputation of the source (if source organization is well-known, well-regarded and frequently in the news) can decrease this skepticism.

While the CUT model has a special section for measuring credibility (it is included in the Contents criterion), it should be realized that the question of credibility goes beyond trustworthiness of the published content. Therefore other criteria in the model are also important to establish credibility. For example: regardless of the published content: how credible can a web page be with a bad web design, without a registered domain (on one of a free-hosting providers) and that is unavailable for most of the time?

Furthermore: credibility is obviously not only dependent on the web page but on all ways of communication and behavior of the company. However a study of these factors would be outside the scope of this paper, therefore we concentrate on which factors affect the credibility of a web page and how it can be improved.

Credibility and relationships building are therefore emphasized in the presentation of the model. However, other factors that are included in the CUT model (such as esthetics, technical characteristics, web page structure etc.) should not be neglected as they can help (or damage) considerably in achieving the above-mentioned objectives.

While web pages are widely recognized by both theorists and practicians as an important and necessary addition to public relations strategy, the practical situation is not so bright. While almost all larger companies have their own corporate web page (90\% of Fortune 500 companies had it already in 1997 (Esrock, 1998)), in practice, both efficient planning and evaluation of web pages are lacking. 
Ha (2000) found that not many organizations employ their sites effectively as part of their public relations program. Similarly Stock (2002) found that many investor relations sites fail to meet basic standards and are not well maintained (with many grammatical errors and infrequent updates of information).

Evaluation of a web-page efficiency is also not common - it was found that most companies do not evaluate the efficiency of their web pages. Even when formal systems of evaluation are in place, they are usually only used to count hits of specific sites. Lack of financial resources for evaluation is one of the frequently cited reasons that limit evaluation (Newland-Hill \& White, 2000). The study of US and international investor relations sites (Breakstone, 2001) came to a similar conclusion - most web pages provide static information, but usually do not provide response mechanism, investor interaction and do not use the site to also gather information about investors.

This is an indicator that this field needs much further research. The presented model is an important addition to this field. It provides a comprehensive framework for evaluation that can highlight the most important deficiencies in the use of web pages. It is also relatively cost-effective as different testing methods can be used for obtaining the final grade.

One of the often-mentioned questions in the PR research is how adept are organizational Web sites at meeting stakeholder information needs (Kent et al., 2003). Therefore the model is structured in such a way to ensure that it includes stakeholders' perspective.

\section{Usability Theory}

Before the CUT model is presented we briefly summarize the main concepts of usability and the methods that can be used for its evaluation.

Usability is a branch of ergonomics science involved in design accommodated for the use of human. (McCormick \& Sanders in Hallahan, 2001).

In the early 1980's usability of computer systems again gained on importance with the penetration of personal computers in everyday life. It gained focus of non-technical scientists (cognitive psychologists, anthropologists, sociologists, philosophers). A new multidisciplinary area of research was developed, the so-called Human-Computer Interaction (HCI). Human-Computer Interaction is a field of research and development, methodology, theory, and practice, with the objective of designing, constructing, and evaluating computer-based interactive systems ... so that people can use them efficiently, effectively, safely, and with satisfaction (Hartson, 1998). At the time a special focus was given to understanding user activities in the real world. New methods for usability research emerged. They formed a new discipline - Usability Engineering (also Usability Testing). In the mid 90's the Internet was becoming an important medium and it has given $\mathrm{HCI}$ a new wider dimension.

$\mathrm{HCI}$ is cross disciplinary in its conduct and multidisciplinary in its roots, drawing on synthesizing and adapting from several other fields (Hartson, 1998). Today human-computer interaction combines different scientific disciplines such as electronic engineering, computer science, informatics, cognitive and behavioristic psychology, psychometrics, ergonomics, linguistics, sociology, graphic design, typography (Downtown, 1993).

Although usability engineering is particularly focused on usability, it must be made clear that usability is a relatively narrow field in comparison to system acceptability. A system should satisfy both users' needs and the needs of systems owners (and other stakeholders). However in this paper we mainly concentrate on the needs of the users - therefore in the CUT model web pages are evaluated from the users' perspective. 
Nielsen introduced a system acceptability model (Nielsen, 1993). This model proposes that a system is accepted if it is both socially and practically acceptable. Practical acceptability is defined by usefulness, costs, compatibility and reliability. Usefulness is dependent on utility and usability.

Nielsen defines usability as a multidimensional property of a user interface, usually related to five attributes: learnability, efficiency, memorability, errors and satisfaction (Nielsen, 1993). Rosson and Carroll (2002) define usability with three different perspectives: human performance, learning and cognition, and collaborative activity. In its core this is coherent with Nielsen's. International Standards Organization defines usability as an extent to which in given circumstances a typical user can use a system with satisfaction in order to efficiently and effectively achieve defined goals (ISO, 1994). They define usability wider than Nielsen. Beside satisfaction and efficacy it also includes efficiency.

Both theory and practice attribute a special importance to usability. Usability improves competitiveness, user satisfaction, image of an organization, user productivity (buyers, employees etc), reduces development times, development costs, maintenance costs, training costs, lowers error rates etc. Wider defined usefulness is also harder to evaluate. Therefore the focus of research is mainly on usability.

Several methods for usability testing emerged since the introduction of usability engineering. Most of them emphasize the importance of the so-called minimax principle, that is maximizing a value of information and/or service with given resources or minimizing the use of resources to achieve a given level of information and/or service value. Methods can be categorized in different ways. Based on data collecting technique they can be divided into collecting user opinions, heuristic evaluation, laboratory experiments, technical analysis and hybrids. They can also be divided based on the time needed for execution, complexity and costs of use. Methods use different resources (some need experts, users etc.) and means (from paper and pencil to expensive specialized laboratories). They are differently suited for use through the life cycle of web site development. Methods achieve different levels of detail (for example number of revealed usability problems) and are therefore differently suited for use with critical web systems. We are presenting few of most known and used methods. The list is not thorough but it nevertheless offers a list of choices that can be used for web site evaluation.

For web site evaluation focus groups and questionnaires can be used. Examples of questionnaires are QUIS and WAMMI. The Questionnaire for User Interaction Satisfaction - QUIS is a result of a multidisciplinary group of researchers from the University of Maryland. It is used to subjectively measure user satisfaction (Laboratory for automation psychology and decision processes, 2003). Web site Analysis and Measurement Inventory - WAMMI is used for evaluating easiness of use of web sites (Human Factors Research Group, 2003). The diary keeping method is used and the user is fulfilling the questionnaire during his everyday use of a web site. Contextual enquiry is another example of a questionnaire. It is used for better understanding of the context in which a web site will be used. It is expensive, complicated and it takes a long time to complete. It is useful in the first phases of web site development. Questionnaires can also be extended with interviews to gain in-depth knowledge.

In narrow sense (heuristic evaluation) it represents a list of heuristics, which are inspected by an expert. Nielsen in Molich presented a wider definition of heuristic evaluation. By their definition heuristic evaluation is a systematic walkthrough of user interface from usability perspective (Nielsen, 1993). By that definition it includes expert review, guideline checklist and cognitive walkthrough. Heuristic evaluation is one of the most informal methods. It requires only a small number of evaluators. They can be experts in the domain, usability experts or experts in both. They evaluate the interface based on predefined usability principles (heuristics). It is easy to use, 
cheap and can be quickly used. The main weakness of this method lies in its inability to identify unforeseen errors related to users' needs. It can also be used for a competitive analysis.

Guideline checklist (also guideline reviews) is based on prepared models with instructions, principles and criteria for a detailed review of a web site. Models can be derived from different disciplines (graphical design, programming, usability engineering etc). They are prepared by highly trained professionals, but can be used by non-experts. It is rarely used in practice.

Cognitive walkthrough is used for evaluating intuitiveness of a web page. Its main purpose is to determine whether or not a user can complete a task by using cognitive thinking. It is used in a form of detail simulation of a user's process of solving tasks during the interaction. It reveals the relation between a task and steps that must be performed in order to complete that task. The method is used to test learnability especially in the form of exploration. Special forms of cognitive walkthroughs are pluralistic walkthroughs. They are used when double experts (usability experts that are also experts in the domain) are not available. Stakeholders (representative users, developers, usability experts etc.) evaluate one screen of a web site at the time. A special form of pluralistic walkthroughs is storyboards. They can be used without a computer. They are mainly used for the visualization of a web site. A variation of cognitive walkthrough is cognitive walkthrough assessment. It assesses cognitive effort needed for successful use of a prototype. We can use questionnaires such as Subjective Mental Effort Questionnaire - SMEQ or Task Load Index TLX.

Prototypes are a special version of a web site suited for testing and further development. They can be paper or computer based. A special technique used for testing of advanced concepts of interaction is the Wizard of Oz. The use of this technique is especially suitable in case of the lack of resources and for development of advanced interfaces, agents and advisers. An upgraded version of prototypes is participatory evaluation where users are included in the process of production of a prototype.

Task analysis enables identification of activities and mental process that need to be accomplished in order to fulfill a given task.

User testing is based on observation of a user doing defined tasks, accomplishing specified goals on a given web site. It is recommended to perform observation in a controlled environment laboratory. These laboratories can be equipped with video systems and eye tracking systems. The use of video systems is especially recommended with critical systems. The analysis of recorded sessions can take 3- to 10-times the time needed for user testing itself (Nielsen, 1993). The method can be extended with a retroactive testing where a user is asked to review his recorded session and add comments to his work. Thinking aloud method (also thinking aloud protocol) is a special form of user testing. Users are asked to comment their actions while they work on a particular task. With this method additional information can be derived such as why a user is doing something. Verbalization leads to a better understanding of user's actions, simplifies error detection and enables assumption testing. The results are qualitative, analysis is difficult and takes plenty of time. A specific form of thinking aloud protocol is constructive interaction (also codiscovery method). It assumes the use of two users instead of one. Another variation of user testing is efficacy testing. In contrast to thinking aloud method it focuses on quantitative, objective measures of efficacy. It uses different hardware and software. It can be used to measure the time needed for completion, time spent on a particular page, number of mistakes etc.

Observation is a method where analysts observe a user at his/her working place. The analyst should interfere in user's work as little as possible. He may only interrupt the user if understanding of a particular action is of great importance for further understanding. In a controlled environment it is hard to simulate a context of everyday use. On the other hand real life observation can be expensive. Therefore Hartson and Castillo recommend the use of remote usability 
evaluation (Hartson \& Castillo, 1998). Variations of this method are self-reporting logs and journaled sessions. A user is given (e.g. by e-mail) tasks. During the execution of these tasks the user is asked to record any comments or remarks he might have. The method can be improved with the use of special software, which is used for recording the user's screen while she/he completes the tasks.

Psychophysical methods are mainly used for measuring user satisfaction. Usually it is done with questionnaires. In this way only subjective values can be collected. User satisfaction can also be measured with objective measurements such as EEG, widened pupils, pulse rate, blood pressure, level of adrenaline in blood (Nielsen, 1993).

Card sorting is used for categorizing and combining information in a domain of a web site. This information plays a key role in information architecture. Its use is especially recommended when an evaluator is not well familiar with the domain. It is based on the physiologic research of concept and category formation. A variation of this method is closed card sort. With this method a user is given concepts at the lowest level and higher-level categories. It is especially useful for adding content to an existing web site.

Blind voting is used for the analysis of a user's expectation of where a particular element should be located on a given web page. The web page is distracted in a way that texts are not readable or not understandable, icons and pictures are distracted or replaced etc.

Context of use analysis offers a way to gain additional information about a web site, its properties and users or group of users. It is usually performed in a form of a workshop where representatives of stakeholders (designer, planers, owners, users and others) meet.

Consistency inspection is used to find inconsistencies between pages forming a web site and inside each web page itself. Web site is systematically inspected by representatives of development teams. They then look for compromises on how to abolish these inconsistencies. In contrast to consistency inspection where we look for inconsistencies inside a web site standards inspection is used for comparison of a web site against standards. These standards can be particular for the web sites domain, type of web site etc., but they can also be general such as compliance with HTML, CSS and XML standards. Examples of this group are ISO 9241 compliance check and Section 508 of the Rehabilitation Act. Standards can also be less formal. In that case we talk about customs. Nielsen defines characteristics as de facto standard, when they are used by at least 90\% of large web sites (Nielsen, 1999).

All of contemporary web servers offer an event logging option. Data are usually collected in log files or databases and can be used for user analyses, trends analyses, analysis of enter and exit pages, usage patterns, conversion rates, errors tracking, referrers, analysis of users' equipment (type and version of browsers, operating system, screen resolution etc.). The biggest limitation of the event log analysis is that it can only be used to discover what the users do, but not why they do it.

Interconnectivity analysis is a method that uses specialized software to search for links in a web site and their attributes. This software then analyses the results and presents them in a systematic visual way. The results can be used to assess vertical interconnectivity (links between a home page and other pages), horizontal interconnectivity (links between pages at the same level), web interconnectivity (links between websites pages and pages not part of that website) and number of pages between a home page and the chosen page. Measuring impact factor is used to evaluate relative reputation of a given web site in relation to competitive web sites. Ingwersen (1992) suggests that the influence factor is defined as a quotient of all web sites (including those that are not part of the web site) that link to the web site with number of pages constituting the web site. 
Mystery shopping is used to analyze responsiveness of a web site (its owner, author or staff responsible for communication). The value of evaluation depends on reaction time and quality of response.

Accessibility is one of the key criteria that need to be satisfied. Accessibility is verified with accessibility assessment. Accessibility is determined by technical factor (users hardware and software) and human factor (users ability to use a web site). Guidelines such as Web Content Accessibility Guideline WCAG can be used to assess it (Chisholm, Vanderheiden, \& Jacobs, 1999).

Efforts to reduce costs lead to new automated methods for usability evaluation. Tools for automated evaluation can be used to automate a collection of web site usage data (automated collection), identification (automated analysis) and offers possible solutions (automated criticism) to usability problems (Ivory \& Hearst, 2001). Examples include W3C validators, LIFT, WatchFire Bobby, Dreamweaver 508 Accessibility Suite, AnyBrowser and WAVE. Brajnik analyzed 11 automated tools for web site analysis and discovered that they only focus on some of the usability criteria as download time, alt tags for pictures, link validity, HTML validity etc (Brajnik, 2000).

Credibility can be evaluated with one of heuristic methods. It is defined as perceived property of an object, person or information (Fogg, Marshall, Laraki, Osipovich, \& Varma, 2001).

With a number of different methods available for evaluation a question arises which method should we use. Usually analysts prefer the methods that discover more problems, the methods that discover hard to find problems or the methods that discover big problems. A criterion used can be thoroughness, validity and reliability (Andre, Williges, \& Hartson, 1999). Cunliffe suggests that effectiveness of a method should be validated with: simplicity, efficacy, participation and universality (2000).

Due to the imperfection of methods new methods emerge. New methods are characterized in that they upgrade an existing method with the elements of other existing methods. In this way the borders between methods are diminishing.

\section{The CUT model}

For the evaluation of web pages we chose the CUT model. The model is created with multiattribute decomposition. It is a method frequently used in multi-attribute decision-making. With multi-attribute decomposition a complex evaluation is divided into smaller parts (attributes) that are easier to evaluate. Any of the above-mentioned methods or a combination thereof can be used to evaluate criteria of the CUT model, different methods can be used for different attributes. These attributes are then combined in a hierarchical model by using utility functions.

We have chosen this model, because we believe that the results of web sites evaluation cannot be based on simple addition (even if weighted) of attributes values. For example: a web site with good technologic solutions that is qualitative from the usability point of view, cannot be evaluated as good if it does not offer any (qualitative) content relevant to target interest groups. There are also eliminating factors that need to be fulfilled in order to achieve a given level of quality. The CUT model can be used to analyze influence of each individual attribute on the quality of a web site as a whole, to carry out what-if analysis and sensitivity analysis. What-if analysis enables search for cause-consequence relationships between attributes and final assessment. They are especially important because a correction of one problem can lead to problems in other segments of a web site. Sensitivity analysis can be used for resource allocation and setting priorities.

The CUT model consists of three main groups of attributes: Contents, Usability, Technology (these groups give the model its name). (See Figure 1.) 


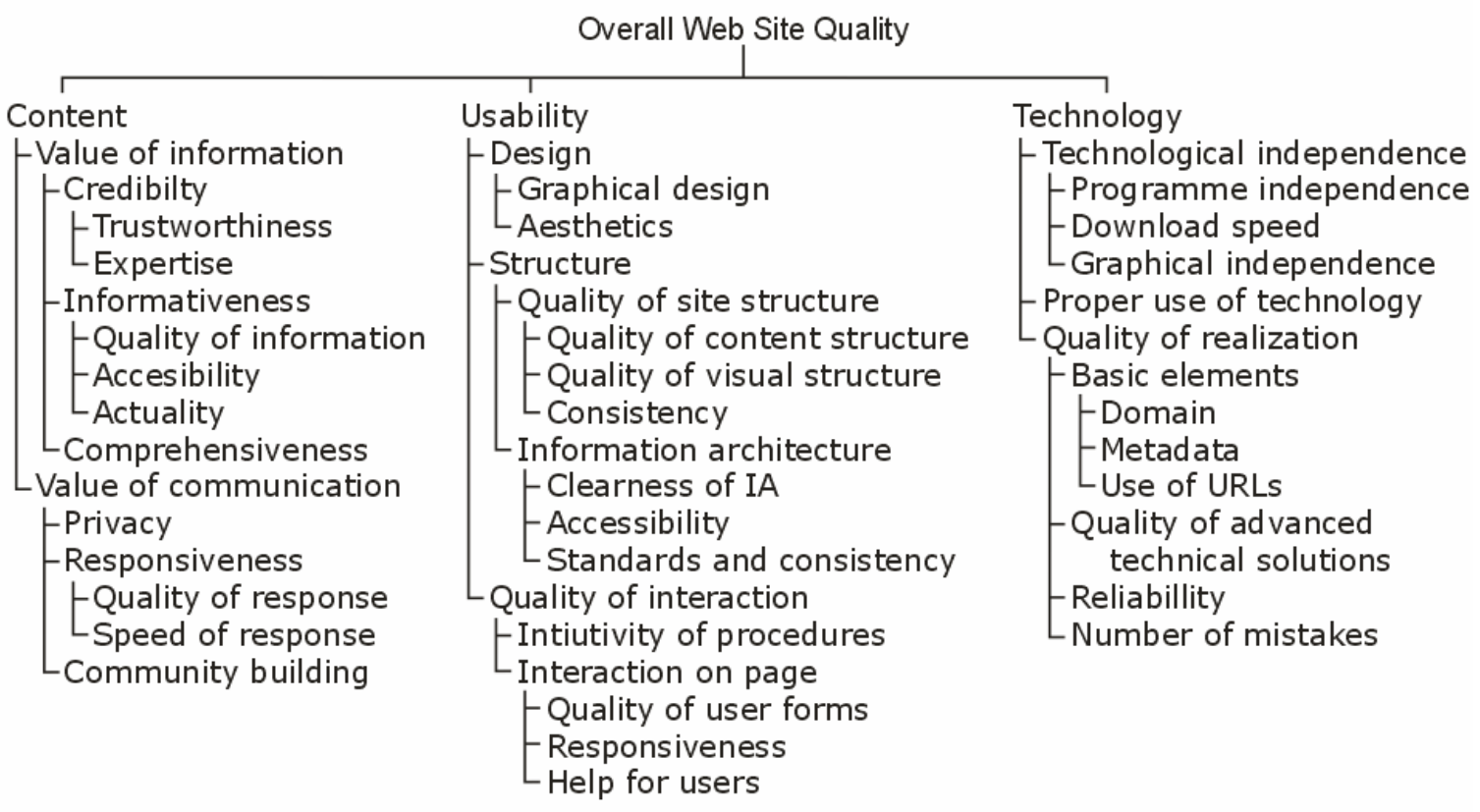

Figure 1: The CUT model

Content is composed of data, information and services. Information can be presented in a variety of ways as a text, picture, video, audio etc. Content is evaluated relatively based on an interest group and its goals. International Academy of Digital Arts and Sciences defines the quality of contents as contents that attracts, is attractive, useful and convenient to targeted user segment (Webby Awards, 2003). The CUT model deals with the evaluation of information and communication separately. Evaluation is evaluated through credibility, informativeness and comprehensiveness. Credibility can be defined as being believable. Tseng and Fogg (1999) present four types of credibility: presumed, reputed, surface and experienced. Surface and experienced credibility can be influenced by an adjustment of a web site (design improvements, usability improvements etc). For value of communication we evaluate privacy, responsiveness and community building. Presumed and reputed credibility can be improved by public relations, marketing etc. Credibility is defined by trustworthiness and expertise. Trustworthiness is perceived goodness or morality of the source. The expertise dimension of credibility captures the perceived knowledge and skill of the source.

Informativeness is quality of information in a broad sense. Its dimensions are quality of information, accessibility and actuality. Information is of quality if it is rich, accurate and in a suitable form. Rich information offers new knowledge. Accurate information describes information that is in accordance with truth. Suitable form is a form adapted to the specifics of World Wide Web, user and his/her culture (including values, beliefs, language). Texts should be short, concise, stereotypes and flourishes should be avoided. Terminology should be selected based on the targeted user segments. If the user is not an expert in the domain, more generic terms should be used. Quality of information can also be improved with the use of innovative, advanced, unusual solutions for a better presentation of known data, information and facts. Information accessibility is defined by rareness of information itself - namely how hard it is to obtain this piece of information. Information actuality is defined by frequency of new contents added and length of time passing between occurrence and publication. Comprehensiveness is defined by the extent of content available on the web site. 
Value of communication is defined by privacy, responsiveness and community building. Privacy is context-dependent property. It is dependent on the type of relationship and the kind of information revealed (Ben-Ze'ev, 2003). In some countries privacy is protected by law. According to research made by Harris Interactive, $90 \%$ of users are concerned about their privacy (Harris Interactive, 2003). Responsiveness measures the quality of messages from web site owners to users. It is a function of the quality of response and the time needed to respond. The value of information decreases with time, therefore the content quality should be higher the longer the response time is. The last attribute related to contents is community building. Communities are among other important for their ability to create contents. Users can perceive these information as more objective, a web site can obtain information that would not be otherwise obtainable or would be expensive to obtain. Examples are chat-rooms, forums, user (customer) support etc.

The next part of the CUT model is related to usability. This part also the most coincides with the usability engineering theory, presented in the previous section. Usability is composed of design, structure and quality of interaction. Design is defined by graphical design and aesthetics factor. The attribute graphical design assesses the extent in which a graphical design supports a strategy of a web site. It has to be consistent with the corporate image or the image of an organization. The chosen colour palette and typography should communicate the identity of trademarks and organization as a whole. Nevertheless colour palettes and typography should be adjusted to the use over the Internet and the targeted user segment. Aesthetics factor has been discussed as inferior (Nielsen, Norman) to usability. However, new researches showed a tight relation between users perception of aesthetics and his/her perception of usability (Tractinsky, Katz, \& Ikar, 2000). It can be measured subjectively. Originality, professionalism and recall level should be evaluated.

Web site structure should be dealt on two levels: the level of a single web page and the level of a web site as a whole. The structure of a web page is made of three attributes: content structure, visual structure and consistency. Content structure deals with partitioning of contents. Is the contents divided in a way that it is intuitive to a user and enables task completion? Visual structure should support content structure. This could be done by positioning elements on a page, using different sizes of elements, using graphical elements (like colours, contrasts, lines, pictures, animated pictures, movies, typographies etc.). Relation between elements can be emphasized by increased distance between these elements. Visual difference can be achieved with space being added between elements. Some researches have been carried out to study user expectation concerning location of different elements (see Bernard, 2000). These expectations can be broken, if a user has no problems realizing that the web site in question follows different standards then it might be expected (see for example Audi's web site case study, Kalbach, 2002). Also consistency should be evaluated: not only visual consistency, but also content and especially system functionality. With achieved consistency users are more confident, they are more prone to exploring and they are looking for new options, possibilities (Lewis in Nielsen, 1993). In this way a web site is more predictable and the possibility of errors decreases.

Information architecture represents the structure of a web site as a whole. It deals with the development of organizational and navigation scheme that allows users effective and efficient movement through a web site (Garrett, 2003). Navigation should effectively communicate relations between possibilities, relations between content and possibilities and users current location. Several different types of navigation exist: global navigation, local navigation, supplementary navigation, contextual navigation, courtesy navigation, breadcrumbs etc. Several auxiliary forms have also been developed such as search, web site maps, indexes etc. Evaluation of information architecture (IA) consists of clearness of IA, accessibility and standards and consistency. Clearness of IA evaluates how well a user understands his options at any time on any page within a web site. A fundamental objective of IA is that a user recognizes an element as means of navigation. Contextual navigation commonly presents a problem in this segment. Accessibility meas- 
ures how hard it is to reach a particular part of a web site. It is one of the attributes with the largest influence on users' efficacy. Contents should be properly categorized, categories and subcategories should be properly named. The results of search queries should also be evaluated. Accessibility can be greatly improved by the use of personalized pages. Last but not least, standards and consistency should be evaluated. If conventions are being followed, a user can routinely perform tasks that he has never performed before. These conventions can be generic to the world wide web (e.g. links should be underlined) or specific to a particular type of a web site (e.g.: groceries are commonly divided into bakery, dairies and produce). If these conventions are being met, a web site is being externally consistent. External consistency can be based on everyday life analogies, but not every principle can be applied to any web site. A web site should also be internally consistent. Web site creators should define these standards. These standards can include nomenclature and graphical elements.

The last dimension of usability based on CUT is interaction quality. Web site interaction is made of procedures intuitiveness and page level interaction. The process of interaction should be as intuitive as possible. This is much more important than the number of steps a user should complete to perform a task. Page level interaction evaluates the quality and responsiveness of web forms. A web form is a program code that is part of a web page and is used to send information from a client (user) to a server. The server then interprets the data, responds, and informs the user of the response. A web form should not be understood solely as a collection of input fields and buttons. It can be any interaction with a web page. Organization of a form and validity of input fields should be checked. It should be checked both on a client and server side if possible. Test such as boundary tests (used to test extreme situations), special (reserved) characters test, maximum number of characters test, empty field test and case sensitiveness test should be performed. Cultural and national differences should also be taken into account, such as: use of currency, number, date and time formats, use of calendars, sorting (the classic example of disregarding national differences is that state abbreviations are obligatory). A user should never lose data he has already put into a form (e.g. because of session timeout). Responsiveness of a web form should be evaluated next. At any time a user should be informed of the status of a process related to a web site. Therefore it is necessary to inform a user of an expected duration of form processing if possible. If that is not possible, a user should be continuously informed of the percent of task being already completed.

To increase the number of successfully completed tasks a web page should be as helpful as possible. Each mistake can lead to a task failure. Mistakes also make users feel uncomfortable, less self-esteemed or even stupid. Mistakes can be avoided by using help. Help should be short, understandable, located near the element that help is provided for. With multilingual versions correctness of translations should be checked.

The last group of attributes is related to technology. Technology is evaluated by using attributes: technological independence, proper use of technology and quality of implementation. Technological independence is evaluated on the basis of technological accessibility. It is a prerequisite to be able to talk about usability and efficiency. Technology independence is built upon program independence, download speed and graphical independence. Program independence is evaluated on the basis of dependability on platform, browser and plug-ins. Evaluation of speed includes both the download time and the time needed to show a web page. It is influenced by three factors: bandwidth, page size and page complexity. Then graphical independence should be evaluated. Special attention should be paid to the choice of screen size (resolution) and colours (not every colour is suitable for use on the web). It should also be checked if a web site could be used without graphics shown.

With attribute proper use of technology we evaluate if advanced technology used is really necessary and if all necessary technologies are used (e.g. secure socket layer for web sites requiring 
high level of security). There is always a time lag between introduction of a new technology and its broad use. We should also check how the use of advanced technology effects the users who are not equipped with this technology.

Last but not least quality of implementation must be evaluated. There are four attributes belonging to the quality of implementation: quality of basic elements, quality of advanced technology solutions, reliability and errors. A reason for separated evaluation of basic elements and advanced technology lies in that the base elements are a precondition while advanced technologies can be used to gain competitive advantage. Base elements are domain, use of metadata and use of URLs. Domain names should be short, easy to remember and should minimize the possibility of typing errors. A proper use of metadata leads to a higher level of findability. URL's should be fixed (because users bookmark web pages, pages can be cited in an offline medium etc.). Because of marketing (e-mail and offline) it is important that URL's are short and simple to enter. Advanced technologies used in this context should not be understood literally. For example audio and video clips, vector (Flash) animations should be evaluated. Case studies for a given technology can be used for benchmarking.

Error is defined as any action that does not lead to completion of a given task (Nielsen, 1993). Errors can be different in their nature and can have different consequences. Some slow down a user, other prevent a user from completing his work and rare methods can even cause tools (e.g. browser or plugin) to stop working.

Reliability can be defined in different ways. It could be the time through which a web site or part of it (e.g. page for transaction processing) is not accessible or not working. It could also be expressed relatively as a percentage of time a web site or part of it is not accessible in a given period (usually in a year). A more sophisticated evaluation would require tests that would analyze a web site to assess if it performs in a constant way.

One of the important advantages of the model is that defined attributes can be evaluated by using different methods. The CUT model is not a priori instructing a use of any method. Each attribute is evaluated in light of a web site goals. All of basic (non derived) attributes have a discrete store of values containing values high, medium (or average) and low. These values are not absolute. They are relatively based. A base can be:

- web site of main offline competitor (or competitors),

- web site of main online competitor (or competitors),

- $\quad$ an aggregate value for a group of web sites (e.g. for a given branch, e-shop, e-banking etc.),

- preceding (earlier) value of the same web site or

- web's best practice (some sort of ideal value).

Several experts (e.g. usability experts, marketing experts, designer, programmers) and users can be included in evaluation. Their number depends on the chosen method, pretentiousness of the method, detail of analysis and size of the evaluated web sites.

\section{Results \& Discussion}

The purpose of this paper was not to perform a comprehensive evaluation of websites. Nevertheless we have chosen 15 web sites. The sample was not completely random. We have chosen web sites of known corporations the shares of which are traded on three different stock exchanges (New York/Nasdaq - USA, Frankfurt/Deutsche Börse - Germany and Ljubljana/ LJSE - Slovenia). A further criterion was the branch of corporations (pharmaceutical, home appliances, brewery, groceries, oil industry). For each stock exchange - branch combination one of the leading companies was chosen. As already mentioned our intention was not to provide an extensive 
evaluation of these sites. We have selected these companies' sites in order to avoid any biases, which might occur as a consequence of the size of a company, its branch, country origin, branch or other factors. The list of selected sites can be found in the appendix.

We used expert review and cognitive walkthrough to evaluate attributes. An exception is a test of responsiveness. For evaluation of responsiveness we used the mystery shopping method. An email with a general question, related to the business of the company was sent. The e-mail was sent on Friday at 4 p.m. local time.

Thorough explanation of measures we used for the validation of individual criteria exceeds the scope of this paper. In accordance with the CUT model we had to analyze expertise and trustworthiness in order to assess credibility. For expertise evaluation we used criteria such as authors' credentials listed, whether there is a list of quotations, any typographical errors and many others. For trustworthiness we used the criteria availability of privacy policy, presence of links to outside materials and sources, is physical address listed, are contact information listed etc. Both criteria were evaluated according to the Prominence-Interpretation Theory (Fogg, 2004). The Prominence-Interpretation Theory suggests that two things happen when people assess credibility: a person notices something (Prominence) and makes a judgment about it (Interpretation). If one or the other does not happen, then there is no credibility assessment. Each criterion was then valued in compliance with a prepared scale. For example trustworthiness was valued high, if all of the criteria used in trustworthiness evaluation were present (privacy policy was present and comprehensible, links to outside materials and sources were available, contact information was listed etc.).

Each of the selected web sites was evaluated by using the CUT model. As shown in Figure 2 none of the web sites evaluated achieved a high overall quality of a web site. On the other hand there were several sites that achieved a high value for contents, usability or technology, but none of them for all criteria.

A further analysis of the results showed two kinds of web sites. A little less than a half have an average $(13 \%)$ or even below average (33\%) value of information. More than a half has a high

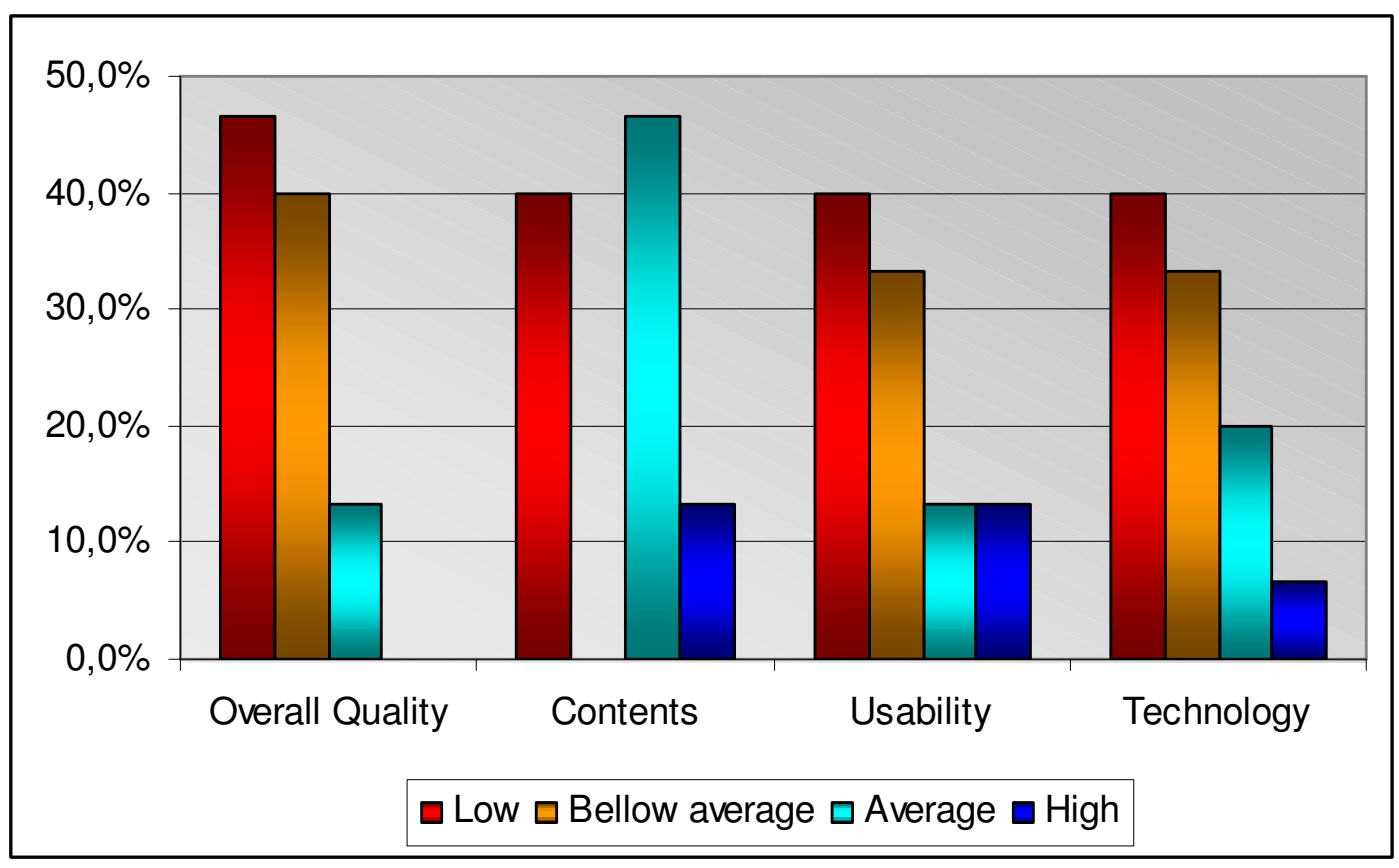

Figure 2: Evaluation Results 
value of information. On the other hand almost half have a low value of communication, with only every fifth having a high value of communication. (See Figure 3.) A low value of communication is mainly a result of poor community building ( $73 \%$ of pages have no form of community building) and poor responsiveness to user questions (47\% with low responsiveness). This shows that most of the analyzed corporations do not realize the possibilities of the Internet as a medium. They still treat the Internet as a classic one-way communication channel (such as

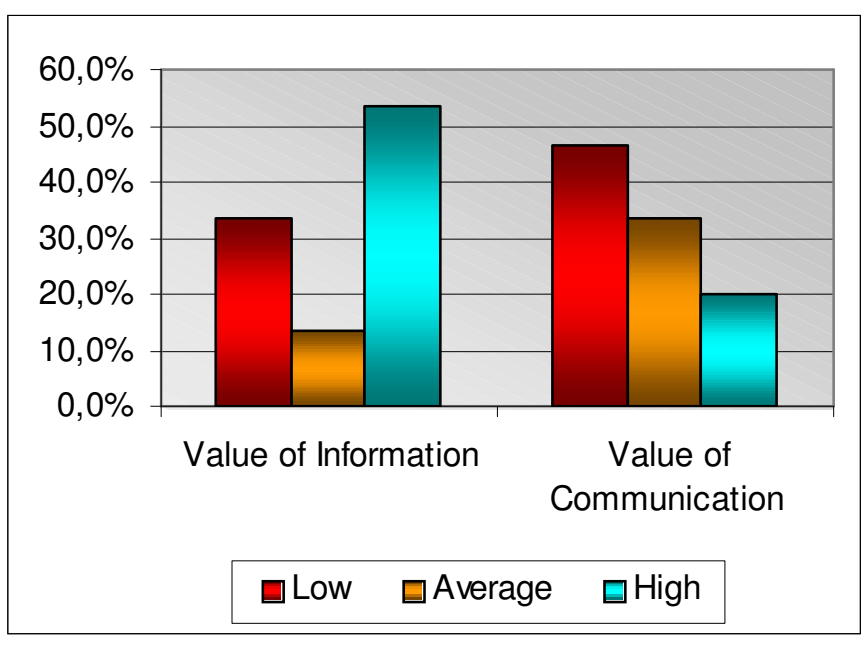

Figure 3: Value of Information and Communication television, radio or newspaper).

However there are already a few companies that try to build a relationship with the user. The classic example is this quote from one of the responses to our mails: "Again, Peter, it was a pleasure to receive your email. Should you have additional questions or comments please contact us. We always enjoy hearing from our friends. Thank you for emailing X. We hope that this information is helpful!"

Although much research has been made in the field of usability, the results for usability criteria are rather poor. Only two of the analyzed web sites have high usability, while four have average usability. As we mentioned in the previous section, evaluation of individual criteria is relative. Most of the analyzed web sites would probably (if they were to retain this level of usability) be evaluated as having a high usability only a few years ago. But an average web site has much improved usability lately. Therefore tolerance levels for usability are a bit higher than they used to be. Low level of usability is mainly a consequence of a poor structure $(53 \%)$ and/or quality of interaction $(40 \%)$.

As described in the previous section, technology is evaluated by using the following criteria: technological independence, proper use of technology and quality of implementation. None of the web sites has a low value for technological independence, with a bit more than a half having an average and a little less high technological independence. The main reason for general low assessments of technology is quality of implementation. $47 \%$ of web sites have a low quality of implementation and one web site had a high value for quality of implementation. The main reasons are the number of mistakes and use of basic elements. 53\% of analyzed web sites had a problem with one of the basic elements (usually metadata or use of URL's). Evaluation of the number of mistakes attribute showed that we only found one web site not having any errors. More than $86 \%$ had several errors. The main reason for this is probably the time pressure under which the launch of a web site is. As already mentioned the lack of resources for formal evaluation can also be an additional reason.

This short study of different pages shows where the main problems and mistakes of a specific site are. Obviously identification of mistakes is crucial (although not enough) for their correction.

The model can also be used for benchmarking studies. 


\section{Conclusion}

As shown in the paper, the importance of the Internet and web pages is on the increase in all areas, especially in public relations. Almost every company (small or large) has its own web page and uses it in one way or another to increase the successfulness of company's operations. However, only a few web pages are properly designed, implemented and evaluated.

Consequently, the need for evaluation of efficiency and successfulness of web pages is also at alltime high and different methods (some of them are also summarized in this paper) can be used for evaluation. Those methods are sometimes fragmented or do not consider all the important factors, needed for a successful web page. Therefore the CUT model is trying to fill this need, as it provides a comprehensive multi-attribute framework for measuring different aspects needed for a quality web page.

The structure of the model results from the fact that all aspects of a web page (content, usability, technology and their sub-categories) have to be efficiently implemented and that the quality of a web page as a whole is not simply the sum of its parts. A severe mistake in one of the main criteria can seriously jeopardize the quality of a web page, even if other factors are excellent. The structure of CUT takes this into consideration.

The other advantage of the presented model is its adaptability. As shown in the paper, the model can be used for the evaluation of companies or other organizations (non-profit organizations, governmental or even personal web pages) from different countries, branches and independently of the size of organization. The relative definition of all values is crucial for this comparison of different web pages.

Since different methods can be used for the evaluation of attributes, the method can also easily be used for both quick (low budget) and extensive (high budget) evaluations. The total cost, time and effort needed for the evaluation obviously depends on the selected method.

However, it should not be forgotten that a web page is "only" an integral part of the whole public relations program of a company. Even the best-designed and maintained web page is fruitless without synergies with other company's efforts in that area. The main concepts of public relations theory and practice from the "old world" (summarized in the first section of this paper) still hold on the World Wide Web. Smart companies can, however, use web pages as a tool for fulfilling and upgrading those principles in order to achieve or maintain their competitive advantage.

\section{References}

A Dictionary of Computing. (2003) "heuristic". Oxford Reference Online. Retrieved June, 52003 from http://www.oxfordreference.com/views/ENTRY.html?subview=Main\&entry=t11.002361

Andre, T., Williges, R., \& Hartson, R. (1999). Effectiveness of usability evaluation methods: Determining the appropriate criteria. Proceedings of Human Factors and Ergonomics Society 43rd Annual Meeting, 1090-1094.

Ben-Ze'ev, A. (2003). Privacy, emotional closeness, and openness in cyberspace. Computers in Human Behavior, 19, 451-467.

Bernard, M. (2000). Examining user expectations of the location of Web objects. Wichita State University. Retrieved from http://www.sandia.gov/itg/newsletter/dec00/article_cui.html

Brajnik, G. (2000). Automatic web usability evaluation: What needs to be done? Proceedings of 6th $\mathrm{Hu}$ man Factors and the Web Conference. Retrieved from http://www.dimi.uniud.it/ giorgio/papers/hfweb00.html

Breakstone, K. (2001). Strategic investor relations--Does it exist? Strategic Investor Relations, 1 (4), 22-28. 


\section{Evaluation of Web Pages as a Tool in Public Relations}

Chisholm, W., Vanderheiden, G., \& Jacobs, I. (1999). Web content accessibility guidelines 1.0. W3C. Re trieved from http://www.w3.org/TR/WAI-WEBCONTENT/

Cotterman W, \& Kumar K. (1989). User cube: A taxonomy of end users. Communications of the ACM, 32 (11), 1313-1320.

Cunliffe, D. (2000). Developing usable Web sites - a review and model. Internet Research: Electronic Networking Applications and Policy, 10 (1), 295-307.

Cutlip, S., Center, A., \& Broom, G. (1994). Effective public relations (7th ed.). Englewood Cliffs, NJ: Prentice Hall.

Downtown, A. (1993) Engineering the human-computer interface. London: McGraw-Hill, 13-27.

Esrock, S., \& Leichty, G. (1998). Social responsibility and corporate Web Pages: Self-presentation or agenda-setting? Public Relations Review, 24 (3), 305-319.

Esrock, S., \& Leichty, G. (1999). Corporate World Wide Web pages: Serving the news media and other publics. Journalism and Mass Communication Quarterly, 76 (3), 456-467.

Esrock, S., \& Leichty G. (2000). Organization of corporate Web pages: Publics and functions. Public Relations Review, 26 (3), 327-344.

Fogg, B. J., Marshall, J., Laraki, O., Osipovich, A., Varma, C. et. al. (2001). What makes Web sites credible? CHI, 3 (1), 61-68.

Fogg, B. J. (2002). Prominence-interpretation theory: Explaining how people assess credibility. A research report from the Stanford Persuasive Technology Lab, Stanford University. Retrieved December, 10 2003 from http://credibility.stanford.edu/pit.html

Garrett, J. (2003). The elements of user experience, user-centered design for the Web. New York: New Riders.

Grunig, J. E., \& Hunt, T. (1984). Managing public relations. New York: Holt Reinhart and Winston.

Ha, L., \& Pratt, C. B. (2000). The real state of public relations on the World Wide Web. The Public Relations Strategist, 6 (3), 30-33.

Hachigian, D., \& Hallahan, K. (2003). Perception of public relations web sites by computer industry journalists. Public Relations Review, 29, 43-63.

Hallahan, K. (2001). Improving public relations web sites through usability research. Public Relations Review, 27 (3), 223-239.

Harris Interactive. (2003). Different Levels of Concern with Privacy. eMarketer. Retrieved March, 272003 from http://www.emarketer.com/news/article.php?1002142

Hartson, R. (1998). Human-computer interaction: Interdisciplinary roots and trends. The Journal of Systems and Software, 43, 103-118.

Hartson, R., \& Castillo J. (1998). Remote evaluation for post-deployment usability improvement. Proceedings of AVI '98 (Advanced Visual Interfaces). pp. 22-29.

Human Factors Research Group. (2003). What is SUMI? Retrieved June, 102003 from http://www.ucc.ie/hfrg/questionnaires/sumi/whatis.html

Ingwersen, P. (1998). The calculation of web impact factors. Journal of Documentation, 54 (2), 236-243.

ISO - International Standards Organization. (1994). ISO Norm 9421: Ergonomic requirements for office work with visual display terminals, part 11 (Guidance on usability).

Ivory, M. \&, Hearst, M. (2001). State of the art in automating usability evaluation of user interfaces. ACM Computing Surveys, 33 (4), 470-516.

Kent, M., \& Taylor, M. (1998). Building dialogic relationships through the World Wide Web. Public Relations Review, 24 (3), 321-334. 
Kent, M., Taylor, M., \& White, W. (2003). The relationships between Web site design and organizational responsiveness to stakeholders. Public Relations Review, 29, 63-77.

Laboratory for automation psychology and decision processes. (2003) QUIS. University of Maryland. Retrieved June, 62003 from http://lap.umd.edu/QUIS/

Middleberg, D, \& Ross, S. (1999). Media in Cyberspace Study—1999. Sixth Annual National Survey. New York: Middleberg+Associates.

Middleberg, D., \& Ross, S. (2000). Survey of media in the wired world -2000. Seventh Annual National Survey. New York: Middleberg+ Associates.

Newland-Hill, L., \& White, C. (2000). Public relations practitioners' perception of the World Web as a communications tool. Public Relations Review, 26 (1), 31-51.

Nielsen, J. (1993). Usability engineering. San Francisco: Morgan Kaufmann.

Nielsen, J. (1999). When bad design elements become the standard. Jakob Nielsen's Alertbox, http://www.useit.com/alertbox/991114.html

Rosson, M. B., \& Carroll, J. M. (2002). Usability engineering, scenario-based development of humancomputer interaction. San Francisco: Morgan Kaufmann.

Silverstein, L. D. (1987). Human factors for color display systems: Concepts, methods, and research. Color and the computer. New York: Academic Press, 38-51.

Stock, H. (2000). IR Web site users say some leave a lot to be desired. Investor Relations Business, November 4 .

Taylor, M., Kent, M., \& White, W. (2001). How activist organizations are using the Internet to build relationships. Public Relations Review, 27 (3), 263-284.

Tractinsky, N., Katz, A. S., \& Ikar, D. (2000). What is beautiful is usable. Interacting with Computers, 13, 127-145.

Tseng, H., \& Fogg, B. J. (1999) Credibility and computing technology. Communications of the ACM, 42 (5), 39-44.

Webby Awards: Judging: Criteria. (2003). Retrieved February, 2, 2003 from http://www.webbyawards.com/main/webby_awards/criteria.html

White, C., \& Raman, N. (2000). The World Wide Web as a public relations medium: The use of research, planning and evaluation in Web site development. Public Relations Review, 25 (4), 405-419.

\section{Appendix}

The following sites were used for testing the CUT model:

\section{USA}

Merck http://www.merck.com/ pharmaceutical company

Anheuser-Busch http://www.anheuser-busch.com/ brewery

ExxonMobil http://www.exxonmobil.com/ oil company

Whirlpool http://www.whirlpool.com/ home appliances

Wal-Mart http://www.walmart.com/ retailing chain

\section{EUROPE}

Schering http://www.schering.de/ pharmaceutical company

Heineken http://www.heinekeninternational.com/ brewery 
$\mathrm{BP}$

http://www.bp.com/

oil company

Electrolux http://www.electrolux.com/

home appliances

Interspar http://www.spar-int.com/

retailing chain

\section{SLOVENIA}

\begin{tabular}{lll} 
Krka & $\underline{\text { htp://www.krka.si/ }}$ & pharmaceutical company \\
Laško & $\underline{\text { http://www.pivo-lasko.si/ }}$ & \multicolumn{1}{c}{ brewery } \\
Petrol & $\underline{\text { http://www.petrol.si/ }}$ & oil company \\
Gorenje & $\underline{\text { http://www.gorenjegroup.com/ }}$ & home appliances \\
Mercator & $\underline{\text { http://www.mercator.si/ }}$ & retailing chain
\end{tabular}

\section{Biographies}

Peter Trkman, M. Sc., is an assistant lecturer for Information Management at the Faculty of Economics, University of Ljubljana, Slovenia. In the past eight years he taught computer lab lectures for first-year students at the Faculty and has written several papers about theoretical and practical considerations of computer literacy education. Additionally, he has published research papers on other topics such as strategic use of information technology and web pages, economics of telecommunications and operations research.

Jaka Lindič, M. Sc., is a teaching assistant at the Faculty of Economics, University of Ljubljana, Slovenia. His main research interests include e-business models, usability and overall quality of web sites. He has experience in the development of educational online systems and corporate Web-based Information Systems. 